\title{
DEVELOPMENT OF CGEM TECHNOLOGY FOR ULTRA-LIGHT TRACKING DETECTORS: THE KLOE-2 INNER TRACKER*
}

\author{
A. Balla ${ }^{\mathrm{a}}$, G. Bencivenni ${ }^{\mathrm{a}}$, P. Branchini ${ }^{\mathrm{b}}, \mathrm{A}^{\mathrm{A}}$ Budano $^{\mathrm{b}}$ \\ M. Capodiferro ${ }^{\mathrm{c}}$, S. Cerioni ${ }^{\mathrm{a}}$, P. Ciambrone $^{\mathrm{a}}$, E. Czerwinski $^{\mathrm{e}}$ \\ E. De Lucia ${ }^{\mathrm{a}}$, G. De Robertis ${ }^{\mathrm{d}}$, A. Di Domenico ${ }^{\mathrm{c}}$, D. Domenici ${ }^{\mathrm{a}}$

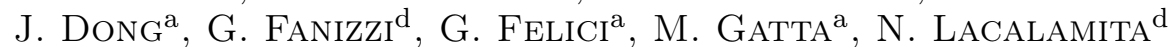

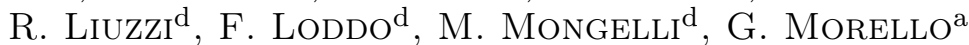

A. Palladino ${ }^{\mathrm{a}}$, A. Pelosi $^{\mathrm{c}}$, L. Quintieri $^{\mathrm{a}}$, A. RAnieri $^{\mathrm{d}}$

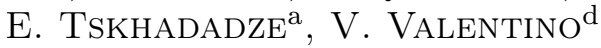

${ }^{a}$ Laboratori Nazionali di Frascati dell'INFN, Frascati, Italy

${ }^{\mathrm{b}}$ INFN Sezione di Roma Tre, Roma, Italy

cDipartimento di Fisica, "Sapienza" Università di Roma

and INFN Sezione di Roma, Roma, Italy

${ }^{\mathrm{d}}$ INFN Sezione di Bari, Bari, Italy

${ }^{\mathrm{e}}$ The Marian Smoluchowski Institute of Physics, Jagiellonian University

Reymonta 4, 30-059 Kraków, Poland

(Received August 6, 2013)

KLOE- 2 at the $\Phi$-factory DA $\Phi$ NE of INFN Laboratori Nazionali di Frascati (LNF), is the first experiment using the GEM technology with a cylindrical geometry (CGEM), developed at the LNF by the Inner Tracker group and within the RD51 Collaboration. Four layers with $26 \div 41 \mathrm{~cm}$ diameters and $L=70 \mathrm{~cm}$, each realized as a Triple-GEM detector, compose this new detector. To produce GEM foils of unprecedented size, the novel single-mask manufacturing procedure has been developed with the TE-MPE-EM CERN group, together with the realization of a novel XV readout pattern. The adopted solutions allowed the total material budget to be kept below $2 \%$ of the radiation length $X_{0}$. The final assembly of the Inner Tracker has been completed on March 14, 2014. The production procedure will be reported together with the validation test results.

DOI:10.5506/APhysPolBSupp.6.1053

PACS numbers: 29.40.Cs, 29.40.Gx

* Presented by E. De Lucia at the Symposium on Applied Nuclear Physics and Innovative Technologies, Kraków, Poland, June 3-6, 2013. 


\section{Introduction}

The KLOE experiment has collected $2.5 \mathrm{fb}^{-1}$ of integrated luminosity between 2001 and 2006 at DA $\Phi$ NE, the $e^{+} e^{-} \Phi$-factory at the INFN Laboratori Nazionali di Frascati, fulfilling a vast program of precision kaon and hadron physics measurements [1]. KLOE-2 represents the continuation of KLOE, at an upgraded DA $\Phi$ NE machine, with a new physics program mainly focused on $K_{\mathrm{S}}, \eta$ and $\eta^{\prime}$ rare decays as well as on kaon interferometry and search for physics beyond the Standard Model [2].

Improving the reconstruction performance for tracks near the Interaction Point (IP) is, therefore, of fundamental importance. To improve the resolution on decay vertices close to the IP, reconstructed from low-momentum charged decay secondaries, a new tracking detector has been recently installed in the free space between the Drift Chamber (DC) inner wall and the beam pipe reducing, therefore, the present track extrapolation length.

The technology of Gas Electron Multiplier (GEM), used so far to equip forward regions of experiments at hadron machines (LHCb, COMPASS, TOTEM experiments) fully exploting their outstanding rate capability (up to $1 \mathrm{MHz} / \mathrm{mm}^{2}$ ) has been adopted. The foreseen radiation flux at the $\mathrm{DA} \Phi \mathrm{NE} e^{+} e^{-}$collider will be well below the GEM limit. Nevertheless, the unique lightness of such detectors will be of utmost importance in KLOE-2 to limit the multiple scattering of low-momentum tracks, photon conversions and $K_{\mathrm{S}}$ regeneration.

\section{KLOE-2 Cylindrical-GEM detector description}

The Inner Tracker (IT) will be inserted inside the existing KLOE Drift Chamber [3]: $3.3 \mathrm{~m}$ lenght and $4 \mathrm{~m}$ diameter with $25 \mathrm{~cm}$ inner radius, filled with $\mathrm{He}: \mathrm{iC}_{4} \mathrm{H}_{10} 90: 10$ gas mixture and providing $\sigma_{r, \phi} \simeq 150 \mu \mathrm{m}$ spatial resolution in the bending plane, $\sigma_{z} \simeq 2 \mathrm{~mm}$ along the beam line, $\simeq 3 \mathrm{~mm}$ on decay vertices inside the DC fiducial volume and $\simeq 4-5 \mathrm{~mm}$ on decay vertices close to the IP. The IT contribution to the overall material budget has to be kept as low as possible to minimize the multiple scattering component of the track momentum resolution and the probability of photon conversions. To this extent a limit on the total IT material budget of $2 \%$ of the radiation length $X_{0}$ has been set. The requirements for the spatial resolutions are: $\sigma_{r \phi} \sim 200 \mu \mathrm{m}$ and $\sigma_{z} \sim 500 \mu \mathrm{m}$.

The adopted solution to fulfill these requirements is the Cylindrical-GEM (CGEM) [4], a Triple-GEM detector composed by five concentric cylindrical electrodes: the cathode, to set the drift field, the 3 GEM foils for the electron multiplication and the anode, acting also as readout circuit (Fig. 1, top-left). The IT is composed by four concentric CGEM layers at radii from $13 \mathrm{~cm}$, to preserve the $K_{\mathrm{S}}-K_{\mathrm{L}}$ quantum interference region, to $20.5 \mathrm{~cm}$ due to the 
constraints from DC inner wall at $25 \mathrm{~cm}$. The total active length for all layers is $70 \mathrm{~cm}$. The anode readout of each CGEM is patterned with longitudinal $\mathrm{X}$ strips with $650 \mu \mathrm{m}$ pitch, interleaved, on the same substrate and at the same level, with pads connected through internal vias to form $\mathrm{V}$ strips at an angle within $25^{\circ} \div 27^{\circ}$ and with $650 \mu \mathrm{m}$ pitch, for a total of about 30000 FEE channels. The insertion of the IT is expected to improve the resolution on vertices close to the IP of about a factor 3 [5].
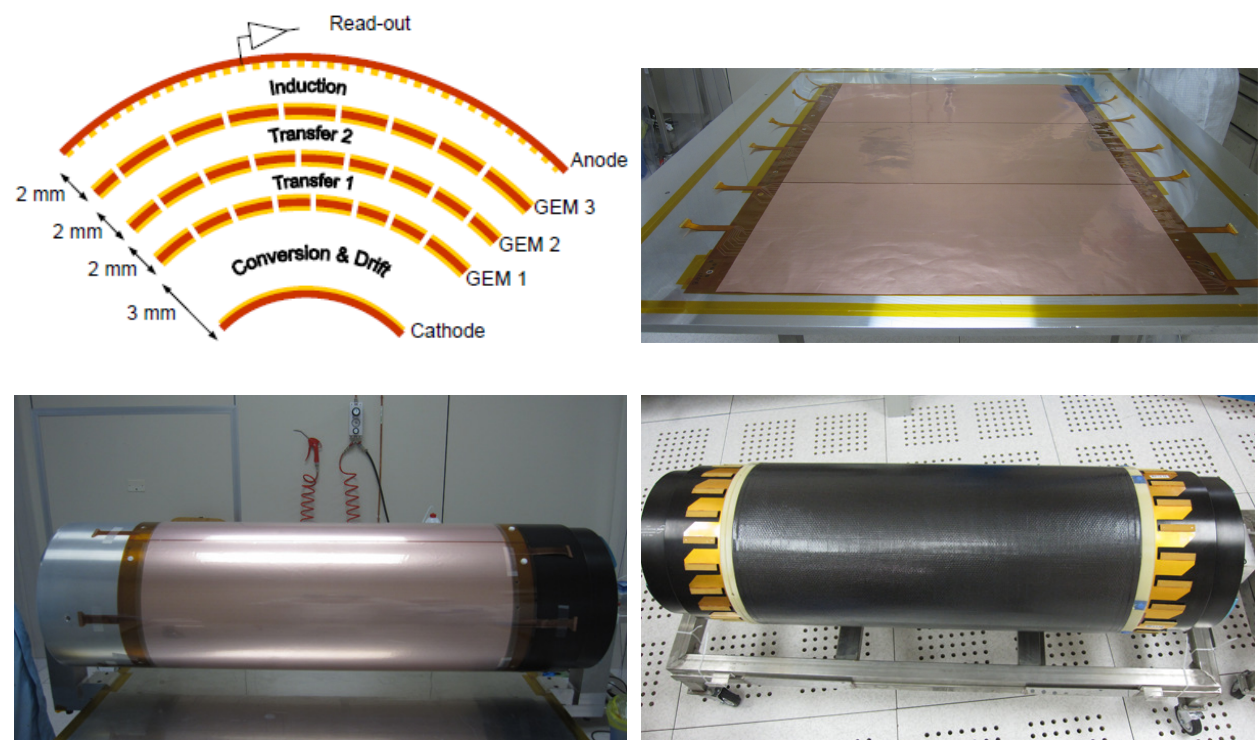

Fig. 1. Top: Cross section of the triple-GEM cylindrical detector (left). The splicing of the three GEM foils (right). Bottom: Gluing the GEM foil on the cylindrical mold (left). The cylindrical readout anode with the carbon-fiber shield (right).

A dedicated readout system has been developed within the KLOE-2 Collaboration. The Front-End Electronics is based on the new GASTONE ASIC [6], a 64 channels chip composed by four different stages: a charge preamplifier with $20 \mathrm{mV} / \mathrm{fC}$ sensitivity, a shaper, a leading-edge discriminator with a programmable threshold, and a monostable stretcher of the digital signal, to synchronize with the KLOE Level-1 trigger. The GASTONE boards are one of the three elements of the IT data acquisition system together with the general interface boards (GIB) and the readout driver (ROD) [7]. The GIB board is based on a Xilinx Virtex 4FX Field Programmable Gate Array (FPGA) with an embedded IBM Power PC (PPC405) running at $300 \mathrm{MHz}$. It has been designed to set up the front-end chip parameters, deliver the power supply and download data from up to eight GASTONE. Data are then delivered from the $2 \mathrm{~Gb} / \mathrm{s}$ Optical port of the 
GIB board to the ROD, which performs a first level event-building. A VME CPU board collects data from the ROD and sends them to the Farm on-line system.

\section{R\&D towards the final Inner Tracker for KLOE-2}

The IT project has been finalized after 3 years of intense $R \& D$, from 2008 to 2010, with several prototypes built and tested with X-rays, cosmicray muons and pion beams at CERN. The three main stages have been: (i) the construction and complete characterization of a full-scale CGEM prototype [8,9]; (ii) the study of the peculiar readout configuration with a planar XV GEM chamber with $40^{\circ}$ stereo angle and its operation in a magnetic field as intense as $1.5 \mathrm{~T}$ at the RD51 CERN-SPS facility [10, 11]; (iii) the construction and characterization of a large area planar GEM realized with the new single-mask photolitografic technique [12, 13]. A spatial resolution $\sigma_{r \phi} \sim 200 \mu \mathrm{m}$ in the bending plane has been measured testing the planar XV GEM chamber with $40^{\circ}$ stereo angle at the 0.52 T KLOE magnetic field value. Vice versa, the magnetic field practically does not affect the second coordinate (in the non-bending plane), providing a spatial resolution of $\sigma_{z} \sim 350 \mu \mathrm{m}$. Due to the total dimesions of the IT, the R\&D included the tuning and the development of a new GEM manufacturing procedure with the TE-MPE-EM CERN group to produce foils of unprecedented size (up to $50 \times 100 \mathrm{~cm}^{2}$ ) with a single-mask electro-chemical etching of the micro-holes. Exploiting the intrinsic lightness of the GEM foils $(50 \mu \mathrm{m}$ thick polyimide doubly clad with $2 \mu \mathrm{m}$ copper films) and their extreme flexibility, we managed to manufacture a cylindrical GEM foil with $2 \mathrm{~mm}$ wide overlap gluing region only. This activity has been followed and supported within the RD51 Collaboration. The gas mixture $\left(\mathrm{Ar} / \mathrm{iC}_{4} \mathrm{H}_{10}\right)$ has been chosen after testing several possible alternatives (either argon or helium based), taking into account the most important operating parameters, including the discharge probability measured with $\alpha$-particles.

\section{Inner Tracker construction}

In the cylindrical Triple-GEM detector, all the five electrodes are made of polyimide foils. The uniformity of the geometrical characteristics of the GEM holes, directly affecting the gain, has been measured over a surface as large as $70 \times 30 \mathrm{~cm}^{2}$. The anode quality control has been perfomed with a 100 ps precision Time Domain Reflectometer [14]: the strip can be regarded as a transmission line and its length and possible damages can be evaluated by measuring the delay of the reflected input signal. 
After the quality controls, three separate GEM foils are spliced together to obtain a single electrode with dimensions of about $100 \times 70 \mathrm{~cm}^{2}$. The splicing is performed by distributing an epoxy glue (Araldite 2011) along the overlap between foils ( $2 \mathrm{~mm}$ wide) and then pressing the foils with a vacuum bag on a machined assembly table, for the 24 hours epoxy curing cycle (Fig. 1, top-right). Then, this large electrode foil is wrapped around an aluminum cylindrical mold, then glued on a $2 \mathrm{~mm}$ wide overlap and finally, enveloped in a vacuum bag, as shown in Fig. 1, bottom-left. Two fiberglass rings are glued at the ends of the barrel, acting as spacers, for the definition of the gas gap between the different electrodes of the detector, and as mechanical support. The surface of the mold is covered by a PTFE film, providing a low-friction surface, allowing the GEM to be extracted without damages. To cope with possible mechanical relaxation of the GEM foil temperature-induced, the two CGEM layers with larger diameter have been built introducing a $300 \mu \mathrm{m}$ thick support grid made of PEEK, a clean organic thermoplastic polymer, between GEM electrodes. The cathode and the anode cylindrical electrodes are manufactured with a similar process, the only difference with respect to the GEM electrodes is that they are both reinforced with a $3 \mathrm{~mm}$ thick honeycomb structure coupled with thin external skins: $50 \mu \mathrm{m}$ kapton foil for the cathode and $90 \mu \mathrm{m}$ carbon foil for the anode (Fig. 1, bottom-right). The assembly of the final detector is performed by inserting one into the other the cylindrical electrodes, starting by the anode and ending with the cathode. To this extent a dedicated machine has been built. The machine allows the insertion of the electrodes with $\mathrm{a} \sim 100 \mu \mathrm{m}$ axial alignment over a length of about $1.5 \mathrm{~m}$. The detector is then sealed at both ends to ensure the gas tightness.

\section{Detector validation tests}

All four CGEM layers of the Inner Tracker have been tested in current mode with $5.9 \mathrm{keV}$ X-rays and equipped with GASTONE FEE. To validate the CGEM layers, cosmic-ray muons data and a $\mathrm{Sr}^{90}$ source have been used. A test stand has been setup at the LNF with final high voltage cables and distribution, final GASTONE FEE boards and signal cables. The DAQ system uses the final GIB boards and ROD. The trigger for data acquisition with cosmic-ray muons is provided by two scintillators, placed above and below the CGEM layer to be tested. To measure cluster efficiencies and spatial resolutions, an external tracking system is provided by three planar tripleGEM chambers (PGEMs) with XY strips readout: one PGEM is placed above and two under the CGEM layer to be tested. The $\mathrm{Sr}^{90}$ source is used to check the CGEM HV sectors, a source scan is performed by positioning the $\mathrm{Sr}^{90}$ on each $\mathrm{HV}$ sector. This allows the total of $\mathrm{X}$-view strips to be 
illuminated while the shortest strips of the V-view are not illuminated, due to the readout geometry. This fast test allows to check the cabling and the uniformity of the detector response. Cosmic-ray muons triggered by the two scintillators are then selected requiring a track reconstructed by the external tracking provided by three planar Triple-GEM chambers. This allowed the cluster efficiency for the two views of the CGEM readout and the spatial resolution in the transverse plane and along the cylinder axis to be measured. Figure 2 shows the distribution of the clusters reconstructed in a CGEM layer in the unrolled anode foil plane $(\rho-\xi), \xi$ along the CGEM axis, and selected requiring a track reconstructed with the three PGEMs. After its integration on DA $\Phi$ NE beam pipe, the Inner Tracker has been tested using a pulsing system and with cosmic-ray muons, to check the on-detector cabling and operational conditions.

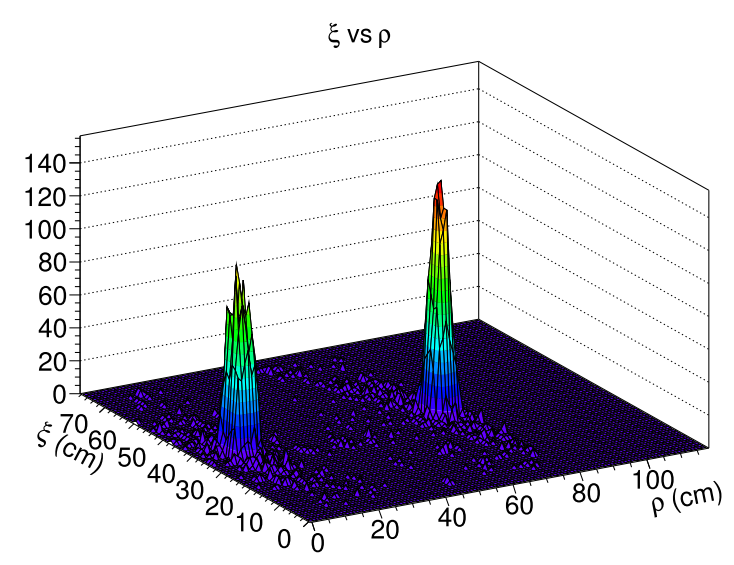

Fig. 2. Clusters reconstructed in a CGEM layer in the unrolled anode foil plane $(\rho-\xi), \xi$ along the CGEM axis, and selected requiring a cosmic-ray muon track reconstructed with the three PGEMs.

\section{Conclusions}

KLOE-2 will be the first experiment in high energy physics to use CGEM detectors. After three years of successful R\&D, which demonstrated the feasibility of a cylindrical triple-GEM detector with an XV readout, the construction of the KLOE-2 Inner Tracker has been completed in March 2013. The detectors have been extensively tested showing a good operational stability and all the expected performance. The Inner Tracker has been mounted on DA $\Phi N E$ beam pipe and inserted inside the KLOE apparatus in June 2013. 


\section{REFERENCES}

[1] F. Bossi et al. [KLOE Collaboration], Riv. Nuovo Cim. 31, 531 (2008).

[2] G. Amelino Camelia et al., Eur. Phys. J. C68, 619 (2010).

[3] M. Adinolfi et al., Nucl. Instrum. Methods A488, 51 (2002).

[4] G. Bencivenni, D. Domenici, Nucl. Instrum. Methods A581, 221 (2007).

[5] F. Archilli et al. [KLOE-2 Collaboration], Technical design report of the inner tracker for the KLOE-2 experiment, arXiv:1002.2572 [physics.ins-det]; LNF-10/3(P) INFN-LNF, Frascati 2010.

[6] A. Balla et al., Nucl. Instrum. Methods A604, 23 (2009); A. Balla et al., Nucl. Instrum. Methods A (2013), http://dx.doi.org/10.1016/j.nima.2013.05.017.

[7] P. Branchini et al., JINST 8, T04004 (2013).

[8] G. Bencivenni et al., Nuclear Symposium Conference Record, 2007 IEEE 6, 4666 (2007).

[9] G. Bencivenni et al., Nuclear Symposium Conference Record, 2008 IEEE, 1345 (2008).

[10] A. Balla et al., Nuclear Symposium Conference Record, 2009 IEEE, 2268 (2009).

[11] A. Balla et al., Nucl. Instrum. Methods A628, 194 (2011).

[12] M. Alfonsi et al., Nucl. Instrum. Methods A617, 151 (2010).

[13] A. Balla et al., Physics Procedia 37, 522 (2012).

[14] G. Bencivenni et al., Nucl. Instrum. Methods A698, 185 (2013). 